\title{
ERGODICITY AND ANNULAR HOMEOMORPHISMS OF THE TORUS
}

\author{
RENATO B. BORTOLATTO AND FABIO A. TAL
}

\begin{abstract}
Let $f: \mathbb{T}^{2} \rightarrow \mathbb{T}^{2}$ be a homeomorphism homotopic to the identity and $F: \mathbb{R}^{2} \rightarrow \mathbb{R}^{2}$ a lift of $f$ such that the rotation set $\rho(F)$ is a line segment of rational slope containing a point in $\mathbb{Q}^{2}$. We prove that if $f$ is ergodic with respect to the Lebesgue measure on the torus and the average rotation vector (with respect to same measure) does not belong to $\mathbb{Q}^{2}$ then some power of $f$ is an annular homeomorphism.
\end{abstract}

\section{INTRODUCTION}

The rotation set is a well known conjugation invariant for homeomorphisms of the torus that are homotopic to the identity. Inspired by the rotation number of Poincaré we can start with such homeomorphism $f$, fix a lift $F$ of $f$ acting on the plane and define the rotation set $\rho(F)$ as the set of accumulation points of the sequences

$$
\left\{\frac{F^{n_{i}}\left(x_{i}\right)-x_{i}}{n_{i}}\right\}_{i \in \mathbb{N}}
$$

where $x_{i} \in \mathbb{R}^{2}$ for all $i \in \mathbb{N}$ and $n_{i} \in \mathbb{N}$ is such that $n_{i} \stackrel{i \rightarrow+\infty}{\longrightarrow}+\infty$.

For a homeomorphism $f$ on the torus homotopic to the identity the limit

$$
\lim _{n \rightarrow \infty} \frac{F^{n}(x)-x}{n},
$$

when it exists, is denoted by $\rho_{p}(F, x)$ and called pointwise rotation vector. We note that, if $\pi: \mathbb{R}^{2} \rightarrow \mathbb{T}^{2}$ is the covering map, then $\rho_{p}(F, x)=\rho_{p}(F, y)$ whenever $\pi(x)=\pi(y)$. In contrast with the rotation number of Poincaré (for orientation preserving homeomorphisms of the circle) it does not need to exist for every $x \in \mathbb{R}^{2}$ and even when it does exist for every $x \in \mathbb{R}^{2}$ the limit can still depend on $x$. Furthermore, $\cup_{x \in \mathbb{R}^{2}} \rho_{p}(F, x)$ is not necessarily equal to $\rho(F)$ as the later can be shown to be convex, unlike the former. For this reason, a vector $v \in \rho(F)$ for which there is $x$ satisfying $\rho_{p}(F, x)=v$ is said to be realized by $\pi(x)$. A source for this and other results is the seminal paper of Misiurewicz and Ziemian [14].

The question of which dynamical properties of $f$ can be deduced from $\rho(F)$ is somewhat well understood, specially when $\rho(F)$ has non-empty interior. For example, when $\rho(F)$ has non-empty interior it's possible to prove that $f$ has strictly positive topological entropy [13. It's also known that for each $(p / s, q / s) \in \operatorname{int}(\rho(F))$ with $p, q, s \in \mathbb{Z}$ there exists $x \in \mathbb{R}^{2}$ such that $F^{s}(x)-x=(p, q)$ (see [8]). There

1991 Mathematics Subject Classification. Primary: 37E30, 37E45; Secondary: 37A25, 37C25. Key words and phrases. Torus homeomorphisms, rotation sets, ergodicity, periodic points.

Instituto de Matemática e estatística. Universidade de São Paulo (IME-USP). First author supported by CNPq - Brasil. Second author supported by CNPq-Brasil, Proc. \#304360/2005-8. 
exists a similar version when $\rho(F)$ is a non-degenerated segment and $f$ preserves area (see [7]).

Our work here is focused on better understanding of the dynamical properties of $f$ when the rotation set has empty interior, particularly when the homeomorphism preserves the natural area measure $\lambda$ on $\mathbb{T}^{2}$, which we will refer to as the Lebesgue measure on the 2-torus or simply "area". Whenever $\lambda$ is invariant by $F$ one can define, as in 14, the average rotation vector with respect to $\lambda$ as

$$
\rho_{\lambda}(F)=\int_{x \in \mathbb{T}^{2}}\left(F\left(\pi^{-1}(x)\right)-\pi^{-1}(x)\right) d \lambda
$$

We are particularly interested in understanding when the rotation set of $F$ prevents $f$ from having a strictly toral behavior (as defined in [10]). In this note we prove the following theorem.

Theorem 1. Let $f$ be a homeomorphism of $\mathbb{T}^{2}$ homotopic to the identity and $F$ be a lift of $f$ such that $\rho(F)$ is a non-degenerated line segment with rational slope that intercepts $\mathbb{Q}^{2}$. If the Lebesgue measure on the torus is $F$-invariant and ergodic and $\rho_{\lambda}(F)$ does not belong to $\mathbb{Q}^{2}$ then some power of $f$ is an annular homeomorphism.

Here, after [10], by annular homeomorphism we mean that there exists $M>0$, $v \in\left(\mathbb{Z}^{2} \backslash\{(0,0)\}\right)$ and a lift $G$ of $f$ such that

$$
-M \leq\left\langle G^{n}(x)-x, \frac{v}{\|v\|}\right\rangle \leq M, \quad \forall x \in \mathbb{R}^{2}, \forall n \in \mathbb{Z}
$$

were $\|\cdot\|$ denotes the Euclidean norm for $\mathbb{R}^{2}$.

In [6] it is shown that if $\rho(F)$ is a (non-degenerated) line segment and there exists $p \in \mathbb{Q}^{2} \cap \rho(F)$ which is not realized by a periodic orbit then some power of $F$ is annular. In Theorem 1 we require that the Lebesgue measure is ergodic and $F$-invariant, so [7] ensures that any point in $\mathbb{Q}^{2} \cap \rho(F)$ is realized by a periodic orbit.

We emphasize that in [11] the case where $\rho(F)=\{(0,0)\}$ is studied and it is shown that $f$ needs not to be annular, hence the hypothesis on the non-degeneracy of $\rho(F)$ into a point cannot be removed.

It is not simple to exhibit an explicit example of homeomorphism that satisfies the hypothesis of Theorem 1, so let us argue that these hypothesis are in fact very common. Firstly, let $F(x, y)=(x, y+\psi(x))$ where $\psi$ is any 1-periodic, continuous function such that $\int_{0}^{1} \psi(s) d s=\sqrt{2}$, and $\psi(0)=0$. Note that $F$ is a lift of a homeomorphism $f$ of the 2-torus that is homotopic to the identity and preserves area. Additionally, it's easy to see that $\rho(F)$ is a non-degenerated line segment and that $\rho_{\lambda}(F)=(0, \sqrt{2})$.

Now, let $\mathcal{H}_{0}\left(\mathbb{T}^{2}, \lambda\right)$ be the set of homeomorphisms of the 2-torus which are homotopic to the identity, preserve the Lebesgue measure and, for a given $v \in \mathbb{R}^{2}$, let $\mathcal{H}_{0}^{v}\left(\mathbb{T}^{2}, \lambda\right)$ be the subset of $\mathcal{H}_{0}\left(\mathbb{T}^{2}, \lambda\right)$ of homeomorphisms for which there exists a lift such that the average rotation vector with respect to $\lambda$ is $v$. The main result of [4], an extension of the celebrated Oxtoby-Ulam Theorem, implies that ergodicity of the Lebesgue measure is a typical (dense $G_{\delta}$ ) property in both these spaces.

The stated result, however, is still not sufficient for our purposes, but a careful look at the proofs of both Proposition 3 and Theorem 2 of [4] shows that they prove something more. In fact, if $I^{2}=[0,1] \times[0,1]$ and $\pi$ is the described map $\phi$ in Proposition 1 of 4 , their technique show that for every neighborhood $V$ of a 
given $f \in \mathcal{H}_{0}^{v}\left(\mathbb{T}^{2}, \lambda\right)$, there exists an homeomorphism $h$ such that, if $g=h f$ then $g$ is both ergodic and belongs to $V$ and such that $h$ pointwise fixes any point in $\pi\left(\partial I^{2}\right)$. In particular, for the $F$ we took above we have that $F$ fixes pointwise the line $\left\{x \in \mathbb{R}^{2} \mid(x)_{1}=0\right\}$. We can then find $g$ ergodic and with a lift $G$ having the same average rotation vector as $F$, and such that $F$ and $G$ coincide on $\partial I^{2}$. Then one can show that $\rho(G)=\{0\} \times[a, b]$, with $a \leq 0$ and $b \geq \sqrt{2}$, as $G$ has fixed points and $\rho_{\lambda}(G)=\rho_{\lambda}(F)=(0, \sqrt{2})$.

The technique we use in the proof of Theorem 1 is to study the sets $B_{0}, B_{\pi}, \omega\left(B_{0}\right)$ and $\omega\left(B_{\pi}\right)$ as defined in [1, 2]. The presence of these sets, which we'll describe in the next section, have many important dynamical consequences that have proven useful in obtaining a number of results (see for instance [15, 3], 6]). Many ideas used in our proof here follow from 15 .

\section{Preliminaries}

In this work we consider $\mathbb{T}^{2}=\mathbb{R}^{2} / \mathbb{Z}^{2}$ to be the flat 2-torus, and $\lambda$ its area measure, which we call the Lebesgue measure. The covering projection from the universal cover $\mathbb{R}^{2}$ to $\mathbb{T}^{2}$ is denoted by $\pi$. Given a point $x \in \mathbb{R}^{2}$ and the canonical basis, we denote by $(x)_{1}$ (respectively $\left.(x)_{2}\right)$ its first (resp. second) coordinate of $x$. As noted before, we denote by $\mathcal{H}_{0}\left(\mathbb{T}^{2}, \lambda\right)$ the set of area preserving homeomorphisms of $\mathbb{T}^{2}$ which are homotopic to the identity.

Let $S \subseteq \mathbb{R}^{2}$ with $S \neq \emptyset$. We will say that $S$ is unbounded rightward if $\sup _{x \in S}(x)_{1}=+\infty$, and we will say that $S$ is unbounded leftward if $\inf _{x \in S}(x)_{1}=$ $-\infty$. If $S$ is either unbounded leftward or rightward we say that $S$ is horizontally unbounded. Otherwise $S$ is horizontally bounded, in which case there are real numbers $a, b$ such that $S$ is contained in

$$
([a,+\infty[\times \mathbb{R}) \cap(]-\infty, b] \times \mathbb{R})=[a, b] \times \mathbb{R}
$$

In this case we call any real number greater than $|b-a|$ a horizontal bound for $S$. Likewise, a non-empty set $S \subseteq \mathbb{R}^{2}$ will be called unbounded upward if $\sup _{x \in S}(x)_{2}=$ $+\infty$ and unbounded downward if $\sup _{x \in S}(x)_{2}=-\infty$. A set $S \subseteq \mathbb{R}^{2}$ will be called vertically unbounded if it is unbounded upward or downward. Again, in this case there are real numbers $a, b$ such that $S$ is contained in $\mathbb{R} \times[a, b]$.

To fix the terminology, given a curve $\gamma:[0,1] \rightarrow \mathbb{R}^{2}$, we denote by $[\gamma]$ its image. By $\lfloor\rfloor:. \mathbb{R} \rightarrow \mathbb{Z}$ we'll mean the usual floor function. Finally, we'll say that a set $S \subset \mathbb{R}^{2}$ separates two sets $L, R \subset \mathbb{R}^{2}$ if $L$ and $R$ are in distinct connected components of the complement $S^{C}$.

The following two results are relevant in our proofs. The first is a theorem of J. Franks we mentioned in the introduction and the second can be deduced from a theorem of G. Atkinson.

Lemma 1 ([7]). Suppose that $f \in \mathcal{H}_{0}\left(\mathbb{T}^{2}, \lambda\right)$ and $F$ is a lift such that $\rho(F)$ is a non-degenerated line segment. Then for every $(p / s, q / s) \in \rho(F)$ with $p, q, s \in \mathbb{Z}$ there exists $x \in \mathbb{R}^{2}$ such that $F^{s}(x)-x=(p, q)$.

Lemma 2 ([5]). Let $M$ be a compact manifold and $f: M \rightarrow M$ be continuous. Let $\mu$ be a Borelian, ergodic probability measure. Let $g: M \rightarrow \mathbb{R}$ be continuous and satisfy

$$
\int_{M} g d \mu=0
$$


If $A$ is a Borel set and $\mu(A)>0$ then for $\mu$-a.e. $x \in A$ there is a sequence $n_{k} \stackrel{k \rightarrow \infty}{\longrightarrow} \infty$ such that

$$
f^{n_{k}}(x) \stackrel{k \rightarrow \infty}{\longrightarrow} x \quad \text { and } \quad \sum_{i=0}^{n_{k}-1} g\left(f^{i}(x)\right) \stackrel{k \rightarrow \infty}{\longrightarrow} 0
$$

Our main result is a direct consequence of the following statement

Theorem 2. Let $f \in \mathcal{H}_{0}\left(\mathbb{T}^{2}, \lambda\right)$ and let $F: \mathbb{R}^{2} \rightarrow \mathbb{R}^{2}$ be a lift of $f$ such that $\rho(F)=\{0\} \times[a, b]$, with $a \leq-1<1 \leq b$. Suppose $f$ is ergodic with respect to the Lebesgue measure $\lambda$ and that $\rho_{\lambda}(F)$ is of the form $(0, \alpha)$ for some $\alpha \in \mathbb{R} \backslash \mathbb{Q}$. Then there is $M>0$ such that $\left|\left(F^{n}(x)-x\right)_{1}\right| \leq M$ for all $n \in \mathbb{Z}, x \in \mathbb{R}$.

Let us show that Theorem 1 follows from Theorem 2. Let $f, g \in \mathcal{H}_{0}\left(\mathbb{T}^{2}, \lambda\right)$ and let $F$ and $G$ be lifts of $f$ and $g$, respectively. Assume $\rho(F)=\{0\} \times[a, b]$ and $\rho(G)$ is a non-degenerated line segment with rational slope that intercepts $\mathbb{Q}^{2}$. Then there is an invertible map $A \in G L(2, \mathbb{Z})$ such that $A \rho(G)$ is a line segment of the form $\left\{\frac{p}{q}\right\} \times[a, b]$. Using lemma 2.4 in [12] we see $A$ as a change of coordinates in the torus (and as such no other property is destroyed by $A$ ). We can then assume, taking $h=(A g)^{q}$ and $H=(A G)^{q}-(p, 0)$, that $\rho(H)=\{0\} \times[a, b]$ (see [14]). Taking a power $n$ of $h$ such that the length of $\rho\left(H^{n}\right)$ is greater than three and changing the lift we can assume that $a \leq-1<1 \leq b$.

\section{Definitions AND First PROperties}

From now on, let $f$ be a homeomorphism of the 2-torus isotopic to the identity, and let $F$ be a lift of $f$. Let $e_{0}=(1,0)$ and $e_{\pi}=(-1,0)$. We define

$$
V_{0}^{+}:=\left\{x \in \mathbb{R}^{2} \mid\left\langle x, e_{0}\right\rangle \geq 0\right\}=\left\{x \in \mathbb{R}^{2} \mid(x)_{1} \geq 0\right\}
$$

and

$$
V_{\pi}^{+}:=\left\{x \in \mathbb{R}^{2} \mid\left\langle x, e_{\pi}\right\rangle \geq 0\right\}=\left\{x \in \mathbb{R}^{2} \mid(x)_{1} \leq 0\right\}
$$

Consider $\mathbb{R}^{2} \cup\{\infty\} \sim S^{2}$ the one-point compactification of $\mathbb{R}^{2}$ and $\widehat{F}$ the homeomorphism induced by $F$ on $S^{2}$ fixing the point in the infinity. The sets $\widehat{V_{0}^{+}}:=V_{0}^{+} \cup\{\infty\}$ and $\widehat{V_{\pi}^{+}}:=V_{\pi}^{+} \cup\{\infty\}$ correspond to $V_{0}^{+}$and $V_{\pi}^{+}$, respectively, on $S^{2}$.

Let $\widehat{B_{0}}$ be the connected component of

$$
\bigcap_{n \leq 0} \widehat{F}^{n}\left(\widehat{V_{0}^{+}}\right)
$$

that contains the point at the infinity. Let $\widehat{B_{\pi}}$ be the connected component of

$$
\bigcap_{n \leq 0} \widehat{F}^{n}\left(\widehat{V_{\pi}^{+}}\right)
$$

that contains the point at the infinity.

Define the sets $B_{0}$ and $B_{\pi}$ in $\mathbb{R}^{2}$ that correspond, respectively, to the sets $\widehat{B_{0}}$ and $\widehat{B_{\pi}}$ on $S^{2}$. To avoid confusion, in this work we'll use the notation $B(x ; \varepsilon)$ for the ball of center $x$ (either in $\mathbb{T}^{2}$ or in $\mathbb{R}^{2}$ ) and radius $\varepsilon \geq 0$.

We'll need the following result.

Lemma 3. Let $F$ be a lift of a homeomorphism $f$ homotopic to the identity with $(0,0) \in \rho(F)$. Then $B_{0}$ and $B_{\pi}$ are not empty. 
Proof. This follows the ideas in [3], but since the context is somewhat different, we include the proof for completeness. We will show that $B_{0}$ is nonempty, the other case is analogous.

First, assume that, for every $M>0$, there exists $x \in \mathbb{R}^{2}$ and $n>0$ such that $\left(F^{n}(x)-x\right)_{1} \geq M+1$. In this case, for every positive $M$ we can find a positive integer $n(M)$ such that $F^{n(M)}\left(V_{\pi}^{+}\right)$intersects $V_{0}^{+}+(M, 0)$. We claim that, if $M>1$, this implies $F^{n(M)}\left(\partial V_{0}^{+}\right)$intersects $\partial V_{0}^{+}+(M, 0)$. If this was not the case, since $F^{n(M)}\left(V_{\pi}^{+}\right)$intersects but does not contain $V_{0}^{+}+(M, 0)$, it would follow that $F^{n(M)}\left(V_{0}^{+}\right) \subset\left(V_{0}^{+}+(M, 0)\right) \subset\left(V_{0}^{+}+(1,0)\right)$. But these inclusions imply that, for every $x \in V_{0}^{+}, \liminf _{i \rightarrow \infty} \frac{\left(F^{i}(x)-x\right)_{1}}{i} \geq \frac{1}{n(M)}$, and since $\pi\left(V_{0}^{+}\right)=\mathbb{T}^{2}$, we would have $\rho(F) \subset V_{0}^{+}+\left(\frac{1}{n(M)}, 0\right)$, a contradiction. Therefore, for every $M>1$ there exists $n(M)$ such that $F^{n(M)}\left(\partial V_{0}^{+}\right) \cap\left(\partial V_{0}^{+}+(M, 0)\right)$ is not empty. The result now follows exactly like lemma 1 of [15].

Assume now that there exists $M>0$ such that, for all $x$ and all positive integers $i$, $\left(F^{i}(x)-x\right)_{1}<M$. Let $K=\bigcup_{i>0} F^{-i}\left(V_{0}^{+}+(M, 0)\right)$ which is a connected unbounded set, satisfying $F^{-1}(K) \subset K$ and $K \subset V_{0}^{+}$. Note also that $K=K+(0,1)$. Now, if $\widehat{K}$ is the corresponding set in $S^{2}$, then $\widehat{F}^{-i}(K)$ is a nested sequence of connected compact sets, all of which containing the infinity. Let $\widehat{K_{\infty}}=\bigcap_{i=0}^{\infty} \widehat{F}^{-i}(\widehat{K})$ be their intersection, and let $K_{\infty}$ the corresponding set in $\mathbb{R}^{2}$.

We claim $K_{\infty}$ is not empty. Otherwise, by compactness, there would be a first integer $n>0$ such that $F^{-n}(K)$ does not intersect the fundamental domain [M, $M+$ $1] \times[0,1]$. Since $F^{-n}(K)$ is invariant by integer vertical translations, it must also be disjoint from the infinite strip $[M, M+1] \times \mathbb{R}$, and as $F^{-n}(K)$ is connected, it would be contained in $V_{0}^{+}+(M+1,0)$. In particular, $F^{-n}\left(V_{0}^{+}+(M, 0)\right) \subset V_{0}^{+}+(M+1,0)$. This implies that, for all $x \in V_{0}^{+}+(M, 0), \liminf _{i \rightarrow \infty} \frac{\left(F^{-i}(x)-x\right)_{1}}{i} \geq \frac{1}{n}$ which again contradicts $\{(0,0)\} \in \rho(F)$.

But then, since $\widehat{K_{\infty}}$ is connected and contains the infinity, every connected component of $K_{\infty}$ is unbounded, and since

$$
K_{\infty}=\bigcap_{l=0}^{\infty}\left(\bigcup_{i=l}^{\infty} F^{-i}\left(V_{0}^{+}+(M, 0)\right)\right)
$$

is a $F$-invariant set and $K_{\infty} \subset V_{0}^{+}$, it follows that $K_{\infty} \subset B_{0}$.

Note that, since $\widehat{V_{0}^{+}}, \widehat{V_{\pi}^{+}}$are closed and $\widehat{F}$ is a homeomorphism, the sets $\widehat{B_{0}}, \widehat{B_{\pi}}$ are closed and therefore the sets $B_{0}, B_{\pi}$ are also closed. The set $B_{0}$ can be seen as the union of all connected closed, unbounded sets $C$ of $\mathbb{R}^{2}$ that satisfy

$$
\left(F^{n}(x)\right)_{1} \geq 0 \forall n \in \mathbb{N}^{+}
$$

for all $x \in C$. There is a analogous characterization for $B_{\pi}$.

We now define the $\omega$-limit of $B_{0}$ as usual by

$$
\omega\left(B_{0}\right):=\bigcap_{i=1}^{\infty} \overline{\bigcup_{j=i}^{\infty} F^{j}\left(B_{0}\right)}=\bigcap_{i=1}^{\infty} F^{i}\left(B_{0}\right)
$$

The sets $\omega\left(B_{0}\right)$ and $\omega\left(B_{\pi}\right)$ are closed and all of its connected components are unbounded (see [15] proposition 1 items 2,3 and proposition 2 items 2,3). Note that $\omega\left(B_{0}\right) \subseteq B_{0}$ (since $\left.F\left(B_{0}\right) \subseteq B_{0}\right)$ and that $\omega\left(B_{\pi}\right) \subseteq B_{\pi}$. It's easy to see that 
$\omega\left(B_{0}\right)$ and $\omega\left(B_{\pi}\right)$ are completely invariant, i.e., that $F^{i}\left(\omega\left(B_{0}\right)\right)=\omega\left(B_{0}\right)$ for all $i \in \mathbb{Z}$. We'll also need the following proposition (see [15] proposition 1 items 3,4 and proposition 2 items 4,5 ). The equality is not covered in [15] but can be deduced from the same arguments.

Proposition 1. The sets $B_{0}^{C}, B_{\pi}^{C}, \omega\left(B_{0}\right)^{C}$ and $\omega\left(B_{\pi}\right)^{C}$ satisfy the following properties.

(1) Each of the sets $B_{0}^{C}, B_{\pi}^{C}, \omega\left(B_{0}\right)^{C}$ and $\omega\left(B_{\pi}\right)^{C}$ has a single connected component.

(2) If $(p, q) \in \mathbb{Z}^{2}$ with $p \geq 0$ then $B_{0}+(p, q) \subseteq B_{0}$ and $B_{\pi}+(-p, q) \subseteq B_{\pi}$. Furthermore, $B_{0}+(0, q)=B_{0}$ and $B_{\pi}+(0, q)=B_{\pi}$ for all $q \in \mathbb{Z}$.

(3) If $(p, q) \in \mathbb{Z}^{2}$ with $p \geq 0$ then $\omega\left(B_{0}\right)+(p, q) \subseteq \omega\left(B_{0}\right)$ and $\omega\left(B_{\pi}\right)+(-p, q) \subseteq$ $\omega\left(B_{\pi}\right)$. Furthermore, $\omega\left(B_{0}\right)+(0, q)=\omega\left(B_{0}\right)$ and $\omega\left(B_{\pi}\right)+(0, q)=\omega\left(B_{\pi}\right)$ for all $q \in \mathbb{Z}$.

Proposition 2. If $\rho(F)=\{0\} \times[a, b]$ then $\omega\left(B_{0}\right)$ and $\omega\left(B_{\pi}\right)$ are both non-empty.

Proof. By corollary 1 in [15] if $\omega\left(B_{0}\right)$ (respectively $\omega\left(B_{\pi}\right)$ ) was empty we would have that $\rho(F) \cap \operatorname{int}\left(V_{0}^{+}\right) \neq \emptyset$ (respectively $\left.\rho(F) \cap \operatorname{int}\left(V_{\pi}^{+}\right) \neq \emptyset\right)$, a contradiction.

With this information we can divide the proof in two proper cases, namely, either $\pi\left(\omega\left(B_{0}\right)\right) \cap \pi\left(\omega\left(B_{\pi}\right)\right)=\emptyset$ or $\pi\left(\omega\left(B_{0}\right)\right) \cap \pi\left(\omega\left(B_{\pi}\right)\right) \neq \emptyset$. In both cases if Theorem 2 isn't true we'd obtain contradictions with properties of the sets $\omega\left(B_{0}\right)$ and $\omega\left(B_{\pi}\right)$.

To achieve this we'll first prepare some statements on what happens when Theorem 2 fails.

\section{Proof of Theorem 2, initial Claims}

The proof of Theorem 2 will be done by contradiction so henceforth we assume that Theorem 2 is not true. Define the set

$$
A:=\left(\omega\left(B_{0}\right) \cup\left(\omega\left(B_{\pi}\right)+(p, q)\right)\right)^{C}
$$

This set depends a priori of $p$ and $q$, but we have in general the following result.

Proposition 3. Let $M$ be a positive real number. Then there is $x_{M} \in \mathbb{R}^{2}$ and $n\left(x_{M}\right) \in \mathbb{Z}$ such that

$$
\left(x_{M}\right)_{1}<-M \quad \text { and } \quad\left(F^{n\left(x_{M}\right)}\left(x_{M}\right)\right)_{1} \geq M
$$

In particular if $(p, q) \in \mathbb{Z}^{2}$ is given we can take $M>0$ such that $x_{M} \in A$.

Proof. By the contradiction hypothesis there exists $x \in \mathbb{R}^{2}$ and $n(x)$ such that $\left|\left(F^{n(x)}(x)-x\right)_{1}\right| \geq 2 M+1$. Assume that $\left(F^{n(x)}(x)-x\right)_{1}>0$ so that $\mid\left(F^{n(x)}(x)-\right.$ $x)_{1} \mid=\left(F^{n(x)}(x)-x\right)_{1}$.

Since $0 \leq(x)_{1}-\left\lfloor(x)_{1}\right\rfloor<1$. The point $x_{0}:=x-\left(\left\lfloor(x)_{1}\right\rfloor+M+1,0\right)$ satisfies

$$
-M-1 \leq\left(x_{0}\right)_{1}<-M
$$

Since $\left(x_{0}\right)_{1}<-M$ we know $x_{0} \notin V_{0}^{+}$so clearly $x_{0} \notin \omega\left(B_{0}\right)$.

But, since $\left(\left\lfloor(x)_{1}\right\rfloor+M+1,0\right) \in \mathbb{Z}^{2}$, we know that

$$
\left(F^{n(x)}\left(x_{0}\right)-x_{0}\right)_{1}=\left(F^{n(x)}(x)-x\right)_{1} \geq 2 M+1
$$

which, in turn, implies that

$$
\left(F^{n(x)}\left(x_{0}\right)\right)_{1} \geq 2 M+1+\left(x_{0}\right)_{1} \geq 2 M+1-M-1=M
$$


Therefore $x_{0}$ is also not in $\left(\omega\left(B_{\pi}\right)+(M-1, q)\right)$ since one of its iterates has first coordinate greater of equal than $M$ (see $\left(^{*}\right)$ in the previous section and recall that $\left.\omega\left(B_{\pi}\right)+(M-1, q) \subseteq V_{\pi}^{+}+(M-1, q)\right)$. Taking in particular $M=p+1$ we conclude that $x_{0} \notin\left(\omega\left(B_{\pi}\right)+(p, q)\right)$ and therefore $x_{0} \in A$.

For the proof in the case $\left(F^{n(x)}(x)-x\right)_{1}<0$ it's enough to define $y:=F^{n(x)}(x)$ so that $\left(F^{-n(x)}(y)-y\right)_{1}>0$.

Proposition 4. All connected components of $\omega\left(B_{0}\right)$ and $\omega\left(B_{\pi}\right)$ are horizontally unbounded.

Proof. We'll show that all connected components of $\omega\left(B_{0}\right)$ and $\omega\left(B_{\pi}\right)$ cannot be vertically unbounded and horizontally bounded. In particular, since we know that these connected components are unbounded, we'll conclude that all connected components of $\omega\left(B_{0}\right)$ and $\omega\left(B_{\pi}\right)$ are horizontally unbounded (note that, since $\omega\left(B_{0}\right) \subseteq B_{0}$, any connected component of $\omega\left(B_{0}\right)$ can only be unbounded rightward, just as any connected component of $\omega\left(B_{\pi}\right)$ can only be unbounded leftward).

Suppose that a connected component $C$ of $\omega\left(B_{0}\right)$ is vertically unbounded and horizontally bounded. Since $A$ is invariant by vertical translation, for $i \in \mathbb{Z}, C+$ $(0, i)$ is also a connected component of $\omega\left(B_{0}\right)$ and is also vertically unbounded and horizontally bounded.

Clearly if $M \geq \sup _{x \in C}\left|(x)_{1}\right|$ then $\cup_{i=-\infty}^{+\infty}(C+(0, i))$ separates the sets $R:=$ $\left\{x \in \mathbb{R}^{2} \mid(x)_{1} \geq M\right\}$ and $L:=\left\{x \in \mathbb{R}^{2} \mid(x)_{1} \leq-M\right\}$. We know that $\omega\left(B_{0}\right)^{C}$ has a single connected component, and since $L \subset\left(V_{0}^{+}\right)^{C} \subset \omega\left(B_{0}\right)^{C}$, it follows that $R \subset \omega\left(B_{0}\right)$.

But the previous proposition implies there is a point of $L \subset \omega\left(B_{0}\right)^{C}$ that has one of its iterates with first coordinate greater or equal than $M+1$ and therefore belongs to $R$. But this is a contradiction since $\omega\left(B_{0}\right)^{C}$ is completely invariant.

The case where $R$ is completely contained in $\omega\left(B_{0}\right)^{C}$ is analogous: proceeding as in the previous proposition take $y:=F^{n(x)}(x)$. The proof for the connected components of $\omega\left(B_{\pi}\right)$ is also analogous.

We will now examine the two different possibilities, first the case where $\pi\left(\omega\left(B_{0}\right)\right) \cap$ $\pi\left(\omega\left(B_{\pi}\right)\right) \neq \emptyset$ and second the case where $\pi\left(\omega\left(B_{0}\right)\right) \cap \pi\left(\omega\left(B_{\pi}\right)\right)=\emptyset$.

\section{The CASE Where $\pi\left(\omega\left(B_{0}\right)\right) \cap \pi\left(\omega\left(B_{\pi}\right)\right) \neq \emptyset$ LeAds to A Contradiction}

In this section we prove that, since we're assuming that Theorem 2 is not true, we cannot have $\pi\left(\omega\left(B_{0}\right)\right) \cap \pi\left(\omega\left(B_{\pi}\right)\right) \neq \emptyset$. We start noticing that if $\pi\left(\omega\left(B_{0}\right)\right) \cap$ $\pi\left(\omega\left(B_{\pi}\right)\right) \neq \emptyset$ then there are $\left(p_{1}, q_{1}\right),\left(p_{2}, q_{2}\right) \in \mathbb{Z}^{2}$ such that

$$
\left(\omega\left(B_{0}\right)+\left(p_{1}, q_{1}\right)\right) \cap\left(\omega\left(B_{\pi}\right)+\left(p_{2}, q_{2}\right)\right) \neq \emptyset
$$

Let $(p, q)=\left(p_{2}-p_{1}, q_{2}-q_{1}\right)$. By the hypothesis there is $z \in \mathbb{R}^{2}$ such that

$$
z \in \omega\left(B_{0}\right) \cap\left(\omega\left(B_{\pi}\right)+(p, q)\right)
$$

We'll need the following results.

Claim 1. If $O$ is a connected component of

$$
A=\left(\omega\left(B_{0}\right) \cup\left(\omega\left(B_{\pi}\right)+(p, q)\right)\right)^{C}
$$

then $O+(0, i)$ is a connected component of $A$ for all $i \in \mathbb{Z}$.

Proof. By proposition 1 we have $A^{C}+(0, i)=A^{C}$ for all $i \in \mathbb{Z}$. This implies that $A+(0, i)=A$ for all $i \in \mathbb{Z}$, which proves the desired result. 


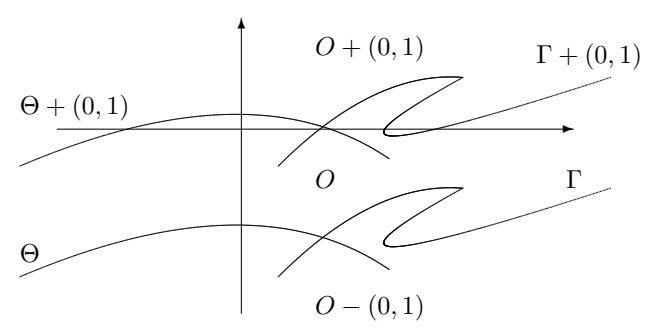

Figure 1. $\Gamma$ and $\Gamma+(0,1)$ are connected components of $\omega\left(B_{0}\right) \subseteq$ $V_{0}^{+}$and $\Theta$ and $\Theta+(0,1)$ are connected components of $\omega\left(B_{\pi}\right)+$ $(p, q) \subseteq V_{\pi}^{+}+(p, q)$. The sets $O, O+(0,1)$ and $O-(0,1)$ are different connected components of $A$

This next claim is illustrated in Figure 1.

Claim 2. Let $O$ be a connected component of $A$ and let $x \in O$. Then $(x+(0, i)) \notin O$ for all $i \in \mathbb{Z}^{*}$. In particular, $O$ and $O+(0, i)$ are distinct connected component of A for all $i \in \mathbb{Z}^{*}$.

Proof. Define, for all $i \in \mathbb{Z}$,

$$
y(i):=x+i(0,1)
$$

Observe that $y(i) \in A$ for all $i \in \mathbb{Z}$. We'll show that every point $y(i)$ must be in a different connected component of $A$.

Suppose this is not the case. In particular, there are $i_{1}<i_{2}$ such that $y\left(i_{1}\right)$ and $y\left(i_{2}\right)$ are in the same connected component of $A$. Let $\gamma$ be a curve connecting $y\left(i_{1}\right)$ and $y\left(i_{2}\right)$ with $[\gamma] \subset A$. Then the curves $\gamma+(0, i)$ also have their images in $A$, for all $i \in \mathbb{Z}$.

The set $\gamma:=\cup_{k \in \mathbb{Z}}\left([\gamma]+k\left(0, i_{2}-i_{1}\right)\right)$ is connected, vertically unbounded (both upward and downward), horizontally bounded and is contained in $A$. Note that $\gamma$ separates the sets $R:=\left\{x \in \mathbb{R}^{2} \mid(x)_{1}>M\right\}$ and $L:=\left\{x \in \mathbb{R}^{2} \mid(x)_{1}<-M\right\}$ for any $M$ greater than the horizontal bound for $\gamma$.

By proposition 4 the connected components of $\omega\left(B_{0}\right)$ and $\omega\left(B_{\pi}\right)$ are horizontally unbounded. Let $z \in \omega\left(B_{0}\right) \cap\left(\omega\left(B_{\pi}\right)+(p, q)\right)$. Let $\Gamma$ be the connected component of $\omega\left(B_{0}\right)$ that contains $z$ and $\Theta$ the connected component of $\omega\left(B_{\pi}\right)+(p, q)$ that contains $z$. Since $\omega\left(B_{0}\right) \subseteq V_{0}^{+}, \omega\left(B_{\pi}\right) \subseteq V_{\pi}^{+}$we conclude that $\Lambda:=\Gamma \cup \Theta$ is connected, unbounded rightward, unbounded leftward and $\Lambda \cap A=\emptyset$, but this leads to a contradiction since $\Lambda \cap \beta \neq \emptyset$.

Since the Lebesgue measure on the torus $\lambda$ is ergodic with respect to $f$, we have that for $\lambda$-almost all $x \in \mathbb{T}^{2}$

$$
\lim _{n \rightarrow \infty} \frac{F^{n} \circ \pi^{-1}(x)-\pi^{-1}(x)}{n}=\int_{x \in \mathbb{T}^{2}}\left(F \circ \pi^{-1}(x)-\pi^{-1}(x)\right) d \lambda=(0, \alpha)
$$

that is, for $\lambda$-almost every point the pointwise rotation vector exists and is of the form $(0, \alpha)$ for some $\alpha$ that we assumed irrational.

Let $x \in A$ and let $O$ be the connected component of $A$ that contains $x$. Since $A$ is open there is $\varepsilon>0$ such that $B(x ; \varepsilon) \subseteq O$. Observe that, for all $\varepsilon>0$ we have 
$\lambda(\pi(B(x ; \varepsilon)))>0$. Let $g:=\left(F \circ \pi^{-1}-\pi^{-1}\right)_{1}$. Then, by Atkinson's lemma, there is a sequence $n_{j} \stackrel{j \rightarrow \infty}{\longrightarrow} \infty$ and $p \in \pi(B(x ; \varepsilon))$ such that for $y \in \pi^{-1}(p)$ we have that $f^{n_{j}}(p) \stackrel{j \rightarrow \infty}{\longrightarrow} p$ and

$$
\left(F^{n_{j}}(y)-y\right)_{1} \stackrel{j \rightarrow \infty}{\longrightarrow} 0
$$

We can assume that $\rho_{p}(F, y)=(0, \alpha)$, since this holds $\lambda$-almost everywhere. Also note that for some $k_{j} \in \mathbb{Z}$ we have $F^{n_{j}}(y) \in\left(B(x ; \varepsilon)+\left(0, k_{j}\right)\right)$. Since $F^{n_{j}}$ is continuous and $A$ is $F$-invariant, $F$ permutes connected components of $A$. By the previous claim

$$
F^{n_{j}}(O)=O+\left(0, k_{j}\right)
$$

which implies that, for all $s \in \mathbb{Z}$,

$$
F^{s n_{j}}(O)=O+s\left(0, k_{j}\right)
$$

Claim 3. There are $j_{1}, j_{2} \in \mathbb{N}$ such that $\frac{k_{j_{1}}}{n_{j_{1}}} \neq \frac{k_{j_{2}}}{n_{j_{2}}}$.

Proof. Assume by contradiction that $\frac{k_{j_{1}}}{n_{j_{1}}}=\frac{k_{j_{m}}}{n_{j_{m}}}$ for all $m \in \mathbb{N}$. We then have

$$
k_{j_{m}}-\varepsilon \leq\left(F^{n_{j_{m}}}(y)-y\right)_{2} \leq k_{j_{m}}+\varepsilon
$$

Dividing by $n_{j_{m}}$ we can take the limit as $m \rightarrow \infty$ to see (since $\rho_{p}(F, y)$ exists) that

$$
\alpha=\lim _{m \rightarrow \infty} \frac{k_{j_{m}}}{n_{j_{m}}}=\frac{k_{j_{1}}}{n_{j_{1}}}
$$

a contradiction, since $\alpha$ is irrational.

Substituting $n_{j_{2}}$ and $n_{j_{1}}$ in (1) we have

$$
O+n_{j_{1}}\left(0, k_{j_{2}}\right)=F^{n_{j_{1}} n_{j_{2}}}(O)=F^{n_{j_{2}} n_{j_{1}}}(O)=O+n_{j_{2}}\left(0, k_{j_{1}}\right)
$$

But this leads to a contradiction: by claim 2 , since $\frac{k_{j_{1}}}{n_{j_{1}}} \neq \frac{k_{j_{2}}}{n_{j_{2}}}$, we must have that $O+n_{j_{1}}\left(0, k_{j_{2}}\right) \neq O+n_{j_{2}}\left(0, k_{j_{1}}\right)$. Therefore, we conclude that the case $\pi\left(\omega\left(B_{0}\right)\right) \cap$ $\pi\left(\omega\left(B_{\pi}\right)\right) \neq \emptyset$ cannot be.

\section{The CASE Where $\pi\left(\omega\left(B_{0}\right)\right) \cap \pi\left(\omega\left(B_{\pi}\right)\right)=\emptyset$ LEAds TO A CONTRADiction}

Since the first possibility lead to a contradiction we examine now the remaining case. It's evident that if $\pi\left(\omega\left(B_{0}\right)\right) \cap \pi\left(\omega\left(B_{\pi}\right)\right) \neq \emptyset$, the distance between these sets is zero. Nevertheless, the same is still true if $\pi\left(\omega\left(B_{0}\right)\right) \cap \pi\left(\omega\left(B_{\pi}\right)\right)=\emptyset$.

Proposition 5. For every $z \in \mathbb{T}^{2}$ and all $\varepsilon>0$, there is a connected set $K \subset \mathbb{R}^{2}$ vertically unbounded (both upward and downward), horizontally bounded and such that for all $y \in K$ there is $n(y) \leq 0$ such that $\pi\left(F^{n(y)}(y)\right) \in B(z ; \varepsilon)$.

Proof. Define $O_{0}=B(x ; \varepsilon)$, where $x \in \pi^{-1}(z)$. As in the previous section we can use Atkinson's lemma to find $n_{j} \in \mathbb{N}^{*}, k_{j} \in \mathbb{Z}$ and $y \in B(x ; \varepsilon)$ such that $F^{n_{j}}(y) \in B(x ; \varepsilon)+\left(0, k_{j}\right)$ and $\rho_{p}(F, y)=(0, \alpha)$. Arguing as is Claim 3 we obtain $j_{1}, j_{2} \in \mathbb{N}$ such that $\frac{k_{j_{1}}}{n_{j_{1}}} \neq \frac{k_{j_{2}}}{n_{j_{2}}}$.

We then define for $n \geq 0$

$$
O_{n}=F^{n_{j_{1}}}\left(O_{n-1}\right) \cup\left(B(x ; \varepsilon)+n\left(0, k_{j_{1}}\right)\right)
$$

Observe that $F^{n_{j_{1}}}\left(O_{0}\right) \cap\left(B(x ; \varepsilon)+\left(0, k_{j_{1}}\right)\right) \neq \emptyset$, so that $O_{1}$ is connected. Since $B(x ; \varepsilon)+\left(0, k_{j_{1}}\right) \subseteq O_{1}$ and $F(x+p, y+q)=F(x, y)+(p, q)$ for all $(p, q) \in \mathbb{Z}^{2}$ we 
have that $F^{n_{j_{1}}}\left(O_{1}\right) \cap\left(B(x ; \varepsilon)+2\left(0, k_{j_{1}}\right)\right) \neq \emptyset$, so that $O_{2}$ is also connected. We see by induction that $O_{n}$ is connected for all $n \in \mathbb{N}$.

Define analogously $V_{0}=B(x ; \varepsilon)$ and for all $n \geq 0$

$$
V_{n}=F^{n_{j_{2}}}\left(V_{n-1}\right) \cup\left(B(x ; \varepsilon)+n\left(0, k_{j_{2}}\right)\right)
$$

Clearly, $V_{n}$ is connected for all $n \in \mathbb{N}$.

We want to see now that $O_{n_{j_{2}}} \cap V_{n_{j_{1}}} \neq \emptyset$. For that note that it follows from the definitions that

$$
O_{n_{j_{2}}} \supseteq F^{n_{j_{1}}}\left(O_{n_{j_{2}}-1}\right) \supseteq F^{2 n_{j_{1}}}\left(O_{n_{j_{2}}-2}\right) \supseteq \ldots \supseteq F^{n_{j_{2}} n_{j_{1}}}(B(x ; \varepsilon))
$$

and

$$
V_{n_{j_{1}}} \supseteq F^{n_{j_{2}}}\left(V_{n_{j_{1}}-1}\right) \supseteq F^{2 n_{j_{2}}}\left(V_{n_{j_{1}}-2}\right) \supseteq \ldots \supseteq F^{n_{j_{1}} n_{j_{2}}}(B(x ; \varepsilon))
$$

We conclude that $O_{n_{j_{2}}} \cup V_{n_{j_{1}}}$ is connected, so it contains the image of a curve $\gamma$ connecting $x+\left(0, n_{j_{2}} k_{j_{1}}\right)$ and $x+\left(0, n_{j_{1}} k_{j_{2}}\right)$. Since $k:=\left|n_{j_{2}} k_{j_{1}}-n_{j_{1}} k_{j_{2}}\right| \neq 0$ the set $K=\cup_{i \in \mathbb{Z}}([\gamma]+i(0, k))$ satisfies the proposition.

The proof of the next claim is similar to the proof of Proposition 9 in 1]. Some of the ideas that follow, especially those concerning figure 2 below, can be traced back to the same paper.

Claim 4. $\overline{\pi\left(\omega\left(B_{0}\right)\right)}=\mathbb{T}^{2}$.

Proof. Assume by contradiction that there is $P \in \mathbb{T}^{2}$ and $\varepsilon>0$ such that $B(P ; \varepsilon) \cap$ $\pi\left(\omega\left(B_{0}\right)\right)=\emptyset$.

By the previous proposition, there is a connected set $K \subset \mathbb{R}^{2}$ such that for all $y \in K$ there is $n(y) \leq 0$ such that $\pi\left(F^{n(y)}(y)\right) \in B(P ; \varepsilon)$. Furthermore $K$ is horizontally bounded and vertically unbounded (both upward and downward), so it separates the sets $R=\left\{y \in \mathbb{R}^{2} \mid(y)_{1}>M\right\}$ and $L=\left\{y \in \mathbb{R}^{2} \mid(y)_{1}<-M\right\}$ for any $M$ greater than the horizontal bound for $K$.

Take $x \in \omega\left(B_{0}\right)$ and let $\Gamma$ be the connected component of $\omega\left(B_{0}\right)$ that passes through $x$. Take $p=M+\left\lfloor(x)_{1}\right\rfloor+1$. Since $p \in \mathbb{Z}$ the set $K+(p, 0)$ also satisfies Proposition 5. Note that $x$ is leftward of $K+(p, 0)$ and recall that $\Gamma$ is unbounded rightward so we have that $\Gamma \cap(K+(0, p)) \neq \emptyset$.

But if $z \in \Gamma \cap(K+(0, p))$ then $z \in \omega\left(B_{0}\right)$ and there is $n(z) \leq 0$ such that $\pi\left(F^{n(z)}(z)\right) \in B(P ; \varepsilon)$. In particular, $F^{n(z)}(z) \in \omega\left(B_{0}\right)$ (since $\omega\left(B_{0}\right)$ is completely invariant) and $\pi\left(F^{n(z)}(z)\right) \in B(P ; \varepsilon)$, a contradiction.

We can show analogously that $\overline{\pi\left(\omega\left(B_{\pi}\right)\right)}=\mathbb{T}^{2}$.

Since $\overline{\pi\left(\omega\left(B_{0}\right)\right)}=\mathbb{T}^{2}$ the set $\pi\left(\omega\left(B_{0}\right)\right)$ must contain at least one non-fixed point and therefore $\omega\left(B_{0}\right)$ must contain a non-fixed point that we'll denote by $x$. By continuity there is a $\varepsilon>0$ such that $F(B(x ; \varepsilon)) \cap B(x ; \varepsilon)=\emptyset$. Since we also have that $\overline{\pi\left(\omega\left(B_{\pi}\right)\right)}=\mathbb{T}^{2}$, there is $(p, q) \in \mathbb{Z}^{2}$ and $y \in \mathbb{R}^{2}$ such that $\|(y+(p, q))-x\|<\varepsilon$ and $y+(p, q) \in \omega\left(B_{\pi}\right)+(p, q)$.

Since $\pi\left(\omega\left(B_{0}\right)\right) \cap \pi\left(\omega\left(B_{\pi}\right)\right)=\emptyset$ the distance between the compact sets $\omega\left(B_{0}\right) \cap$ $\overline{B(x ; \varepsilon)}$ and $\left(\omega\left(B_{\pi}\right)+(p, q)\right) \cap \overline{B(x ; \varepsilon)}$ is a strictly positive number $d \leq 2 \varepsilon$ and is realized by points $x_{1} \in \omega\left(B_{0}\right)$ and $y_{1} \in\left(\omega\left(B_{\pi}\right)+(p, q)\right)$.

Let $v$ be the open line segment connecting $x_{1}$ to $y_{1}$ (see Figure 2). Observe that by our choice of $x_{1}$ and $y_{1}$ we have $F(v) \cap v=\emptyset$ and $v \cap \omega\left(B_{0}\right)=\emptyset=$ $v \cap\left(\omega\left(B_{\pi}\right)+(p, q)\right)$. Let $\Gamma$ be the connected component of $\omega\left(B_{0}\right)$ that contains $x_{1}$ and let $\Theta$ be the connected component of $\omega\left(B_{\pi}\right)+(p, q)$ that contains $y_{1}$.

We know from 15] (Proposition 8) the following fact. 


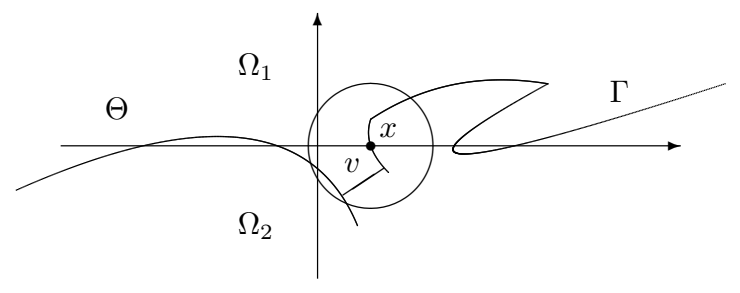

Figure 2. $\Gamma$ denotes a connected component of $\omega\left(B_{0}\right)$ and $\Theta$ denotes a connected component of $\omega\left(B_{\pi}\right)+(p, q)$. The open line segment $v$ links $\Gamma$ and $\Theta$ in an $\varepsilon$-ball centered in $x \in \Gamma$. The set $(\Gamma \cup v \cup \Theta)^{C}$ has two connected components we call $\Omega_{1}$ and $\Omega_{2}$

Claim 5. The set

$$
A=\left(\omega\left(B_{0}\right) \cup\left(\omega\left(B_{\pi}\right)+(p, q)\right)\right)^{C}
$$

has a single connected component. In particular, if $\Gamma$ is a connected component of $\omega\left(B_{0}\right)$ and $\Theta$ is a connected component of $\left(\omega\left(B_{\pi}\right)+(p, q)\right)$ then the set $(\Gamma \cup \Theta)^{C}$ has a single connected component. The set $(\Gamma \cup v \cup \Theta)^{C}$ has exactly two connected components.

The next claim uses an argument similar to the one used in Claim 2.

Claim 6. For all $y \in \mathbb{T}^{2}$ there is $z \in \pi^{-1}(y) \cap A$ and a continuous connected function $\delta: \mathbb{R} \rightarrow A$ such that $\delta(i)=z+(0, i)$ for all integers $i$, and such that $\lim _{|t| \rightarrow \infty}\|\delta(t)\|=\infty$. Furthermore, the image of $\delta$ is a horizontally bounded set $[\delta]$ that separates $\omega\left(B_{0}\right)$ and $\omega\left(B_{\pi}\right)+(p, q)$.

Proof. Since $\pi\left(\omega\left(B_{0}\right)\right) \cap \pi\left(\omega\left(B_{\pi}\right)\right)=\emptyset$ assume, without loss of generality, that $y \notin \pi\left(\omega\left(B_{0}\right)\right)$. Then there is a point $z \in \pi^{-1}(y)$ such that $(z)_{1}>p$. For $i \in \mathbb{Z}$ all points of the form $z+(0, i)$ have first coordinates strictly greater than $p$ and therefore none of them belongs to $\omega\left(B_{\pi}\right)+(p, q)$. In particular, $\{z+(0, i) \mid i \in \mathbb{Z}\} \cap A^{C}=\emptyset$.

By claim 5 the set $A$ has a single connected component hence $z$ and $z+(0,1)$ are in the same connected component of $A$. Consider a curve $\gamma:[0,1] \rightarrow A$ such that $\gamma(0)=z$ and $\gamma(1)=z+(0,1)$. The function $\delta(s):=\gamma(s-\lfloor s\rfloor)+(0,\lfloor s\rfloor)$ has image $[\delta]=\cup_{i \in \mathbb{Z}}([\gamma]+(0, i))$ and satisfies the claim.

Denote by $\Omega_{1}$ and $\Omega_{2}$ the two connected components of $(\Gamma \cup v \cup \Theta)^{C}$. Note that $\Omega_{1}$ and $\Omega_{2}$ are open and that $\partial \Omega_{1}=\partial \Omega_{2}=\Gamma \cup v \cup \Theta$.

Claim 7. Let $y \in \mathbb{T}^{2}$. Take $z \in \pi^{-1}(y)$ and $\delta$ given by the previous claim. Then there is $T>0$ and $k_{1}, k_{2} \in\{0,1\}$ with $k_{1} \neq k_{2}$ such that, for all $t>T, \delta(t) \in \Omega_{k_{1}}$ and for all $t<-T, \delta(t) \in \Omega_{k_{2}}$. In particular, $z+\left(0, j_{1}\right) \in \pi^{-1}(y) \cap \Omega_{k_{1}}$ for all $j_{1}>|T|$ and $z+\left(0, j_{2}\right) \in \pi^{-1}(y) \cap \Omega_{k_{2}}$ for all $j_{2}<-|T|$.

Proof. Since $v$ is bounded and $\lim _{|t| \rightarrow \infty}\|\delta(t)\|=\infty$, there exists $T \in \mathbb{R}$ such that $\delta(t) \cap v=\emptyset$ for all $|t|>T$. Since $[\delta] \subset A$, this implies that for all $t>|T|, \delta(t)$ belongs to either $\Omega_{1}$ or $\Omega_{2}$, and since $\delta$ is continuous there exists $k_{1}, k_{2} \in\{0,1\}$ such that, for all $t>T, \delta(t) \in \Omega_{k_{1}}$ and for all $t<-T, \delta(t) \in \Omega_{k_{2}}$. 
It remains to be shown that $k_{1} \neq k_{2}$. Assume this is not true. Then there is a curve $\beta$ that connects $\delta(-2 T)$ to $\delta(2 T)$ without leaving $\Omega_{k_{1}}$. The set $\left.\delta(]-\infty,-2 T\right) \cup$ $[\beta] \cup \delta(] 2 T,+\infty[)$ is connected, horizontally bounded and vertically unbounded (both upward and downward). By definition, this set does not intercept the set $\Gamma \cup v \cup \Theta$. But this is a contradiction since $\Gamma \cup v \cup \Theta$ is connected and unbounded both leftward and rightward.

Claim 8. $F\left(\Omega_{i}\right) \cap \Omega_{i} \neq \emptyset$ for $i=\{1,2\}$.

Proof. Since $(0,0) \in \rho(F)$ by Lemma 1 there is $y \in \mathbb{T}^{2}$ fixed for $f$ and such that $F(z)-z=(0,0)$ for all $z \in \pi^{-1}(y)$. We conclude therefore that both $\Omega_{1}$ and $\Omega_{2}$ have fixed points, which proves the desired result.

We can now prove the following proposition.

Claim 9. Either $F\left(\overline{\Omega_{1}}\right) \subseteq \overline{\Omega_{1}}$ or $F\left(\overline{\Omega_{2}}\right) \subseteq \overline{\Omega_{2}}$.

Proof. By the definition of $v$ we have that $v \cap \omega\left(B_{0}\right)=\emptyset=v \cap\left(\omega\left(B_{\pi}\right)+(p, q)\right)$. Since $\omega\left(B_{0}\right)$ and $\omega\left(B_{\pi}\right)$ are invariant, $F(v) \cap \Gamma=\emptyset=F(v) \cap \Theta$. Furthermore, $v$ was chosen such that $F(v) \cap v=\emptyset$. Hence,

$$
F(v) \cap(\Gamma \cup v \cup \Theta)=\emptyset
$$

so $F(v)$ is either in $\Omega_{1}$ or in $\Omega_{2}$. Let's assume, without loss of generality, that $F(v) \subseteq \Omega_{1}$. Observe that, since $\Gamma$ is a connected component of $\omega\left(B_{\pi}\right)$ and $\omega\left(B_{\pi}\right)$ is totally invariant, $F(\Gamma)$ is a connected component of $\omega\left(B_{\pi}\right)$. Therefore, either $F(\Gamma)=\Gamma$ or $F(\Gamma) \cap \Gamma=\emptyset$.

In the first case we have that $F(\Gamma) \cap \Omega_{2}=\Gamma \cap \Omega_{2}=\emptyset$. This is also true in the second case: It's clear that $F(\Gamma) \cap \partial \Omega_{1}=\emptyset$ since by the definition of $v$ we have $F(\Gamma) \cap v=\emptyset$ and $\omega\left(B_{0}\right) \cap \omega\left(B_{\pi}\right)=\emptyset$ implies $F(\Gamma) \cap \Theta=\emptyset$. Since $\Gamma \cup v$ is connected $F(\Gamma \cup v)$ is also connected so $F(v) \subseteq \Omega_{1}$ implies $F(\Gamma) \cap \Omega_{2}=\emptyset$. We see analogously that $F(\Theta) \cap \Omega_{2}=\emptyset$. We note that in any case $F\left(\partial \Omega_{1}\right) \cap \partial \Omega_{1}=\emptyset$.

Since $F\left(\partial \Omega_{1}\right) \cap \partial \Omega_{1}=\emptyset$ and $F(v) \subseteq \Omega_{1}$ we have that $F\left(\partial \Omega_{1}\right) \cap \Omega_{2}=\emptyset$. But $\Omega_{1}$ and $\Omega_{2}$ are connected so either $\Omega_{2} \subseteq F\left(\Omega_{1}\right)$ or $\Omega_{2} \cap F\left(\Omega_{1}\right)=\emptyset$.

Assume that $\Omega_{2} \subseteq F\left(\Omega_{1}\right)$ : Then $F^{-1}\left(\Omega_{2}\right) \subseteq \Omega_{1}$, so $F^{-1}\left(\Omega_{2}\right) \cap \Omega_{2}=\emptyset$. But by Claim 7 we know $F\left(\Omega_{2}\right) \cap \Omega_{2} \neq \emptyset$, a contradiction. Therefore we necessarily have that $\Omega_{2} \cap F\left(\Omega_{1}\right)=\emptyset$ and since $F\left(\partial \Omega_{1}\right) \cap \partial \Omega_{1}=\emptyset$ we necessarily must have $F\left(\overline{\Omega_{1}}\right) \subseteq \overline{\Omega_{1}}$.

We are now ready to finish the proof of Theorem 2. There is a small technicality to deal with, however the idea is simple and is illustrated by Figure 3 bellow.

Proof of Theorem 2. Assume that $F\left(\overline{\Omega_{1}}\right) \subseteq \overline{\Omega_{1}}$ (the other case is analogous). Then by induction $F^{s}\left(\overline{\Omega_{1}}\right) \subseteq \overline{\Omega_{1}}$ for $s \in \mathbb{N}$. Since $(0,1),(0,-1) \in \rho(F)$ we know by Lemma 1 that there are $y_{1}, y_{2} \in \mathbb{T}^{2}$ both fixed for $f$ and such that for all $k \in \mathbb{Z}$ we have

$$
F^{k}(z)-z=k(0,1) \quad \text { and } \quad F^{k}(w)-w=k(0,-1)
$$

for all $z \in \pi^{-1}\left(y_{1}\right)$ and $w \in \pi^{-1}\left(y_{2}\right)$.

We can now use Claim 6 putting $y=y_{1}$ to find $z \in \pi^{-1}\left(y_{1}\right)$ and a function $\delta_{1}$ such that $\left[\delta_{1}\right]$ that separates $\omega\left(B_{0}\right)$ and $\omega\left(B_{\pi}\right)+(p, q)$. From Claim 7 we get, without loss of generality, that $z \in \Omega_{1}$. We repeat the same procedure putting $y=y_{2}$ in Claim 6 to find $\delta_{2}$ as before and, by Claim 7, we get, without loss of generality, that $w \in \Omega_{1}$. 


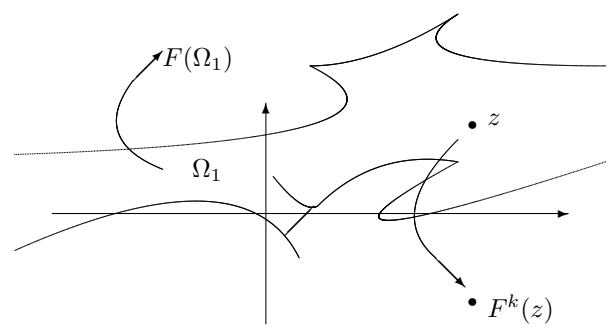

FiguRE 3 . We can't have $F\left(\overline{\Omega_{1}}\right) \subseteq \overline{\Omega_{1}}$ since by [7] there is a point $z \in \mathbb{R}^{2}$ such that $F^{k}(z)-z=k(0,1)$. A similar argument show that the case $F\left(\overline{\Omega_{2}}\right) \subseteq \overline{\Omega_{2}}$ also can't happen

Note that if there is $k>0$ such that either $F^{k}(z)=z+k(0,1)$ or $F^{k}(w)=$ $w+k(0,-1)$ belongs to $\Omega_{2}$ we would have that $F^{k}\left(\overline{\Omega_{1}}\right) \cap \overline{\Omega_{2}} \neq \emptyset$, a contradiction since $F^{s}\left(\overline{\Omega_{1}}\right) \subseteq \overline{\Omega_{1}}$. Therefore, for all $k \geq 0$, both $z+(0, k)$ and $w-(0, k)$ belong to $\Omega_{1}$, and thus, by Claim 7 , there exists $T_{1}>0$ such that, if $k<-T_{1}$, then $z+(0, k) \in \Omega_{2}$.

Let $\beta:[0,1] \rightarrow A$ be a curve joining $z$ and $w$. There exists $L>T_{1}$ such that, if $|k|>L$ then $[\beta]+(0, k)$ is disjoint from $v$, and therefore $[\beta]+(0, k)$ belongs to $\Omega_{1}$ if $k>L$, and to $\Omega_{2}$ if $k<-L$. But this implies that $w-(0, k) \in \Omega_{2}$ for $k<-L$, our final contradiction.

\section{REFERENCES}

[1] S. Addas-Zanata and F. A. Tal, Homeomorphisms of the annulus with a transitive lift, Math. Z., v. 267, p. 971-980, 2011.

[2] S. Addas-Zanata and F. A. Tal, On generic rotationless diffeomorphisms of the annulus, Proc. Am. Math. Soc., v. 138, p. 1023, 2010.

[3] S. Addas-Zanata, F.A. Tal and B.A. Garcia, Dynamics of homeomorphisms of the torus homotopic to Dehn twists, (Arxiv preprint arXiv:1111.5561 v1), 2011.

[4] Alpern, S. and Prasad, V. S., Topological ergodic theory and mean rotation, Proc. Am. Math. Soc. v. 118 (1993), 279-284.

[5] G. Atkinson, Recurrence of co-cycles and random walks, J. London Math Soc. 2, 13 (1976), 486-488.

[6] P. Dávalos, On torus homeomorphisms whose rotation set is an interval, (Arxiv preprint arXiv:1111.2378 1 1), 2011.

[7] J. Franks, The rotation set and periodic points for torus homeomorphisms, "Dynamical systems and chaos.", Aoki, Shiraiwa and Takahashi ed. World Scientific, Singapore, (1995), 41-48.

[8] J. Franks, Realizing rotation vectors for torus homeomorphisms, Trans. Amer. Math. Soc. 311 (1989), no. 1, 107-115.

[9] J. Franks, Recurrence and fixed points for surface homeomorphisms, Ergod. Th. Dynam. Sys. 8 (1988), 99-107.

[10] A. Koropecki and F. A. Tal, Strictly toral dynamics, (Arxiv preprint arXiv:1201.1168v2 ), 2012.

[11] A. Koropecki and F. A. Tal, Area-preserving irrotational diffeomorphisms of the torus with sublinear diffusion (Arxiv preprint arXiv:1206.2409v1), 2012.

[12] A. Kocsard and A. Koropecki, Free curves and periodic points for torus homeomorphisms, Erg. Th. \& Dyn. Sys. (2008), 28, pp. 1895-1915

[13] J. Llibre and R. Mackay, Rotation vectors and entropy for homeomorphisms of the torus isotopic to the identity, Erg. Th. \& Dyn. Sys. 11 (1991) 115-128. 
[14] M. Misiurewicz and K. Ziemian, Rotation sets for maps of Tori, J. London Math. Soc. (2) 40 (1989) 490-506.

[15] F. A. Tal, Transitivity and rotation sets with nonempty interior for homeomorphisms of the 2-torus, Proc. Am. Math. Soc. v. 140 (2012), 3567-3579. 